\title{
Selection Classification for Interaction with Immersive Volumetric Visualizations
}

\author{
Amy Banic \\ University of Wyoming, USA \\ abanic@cs. uwyo.edu
}

\begin{abstract}
Visualization enables scientists to transform data in its raw form to a visual form that will facilitate discoveries and insights. Although there are advantages for displaying inherently 3-dimensional (3D) data in immersive environments, those advantages are hampered by the challenges involved in selecting volumes of that data for exploration or analysis. Selection involves the user identifying a set of points for a specific task. This paper preliminary data collection on natural user actions for volume selection. This paper also presents a research agenda outlining an extension for volume selection classification, as well as challenges, for designing components for a direct selection of volumes of data points.
\end{abstract}

Keywords: HCI methods and theories, Human Centered Design and User Centered Design, Interaction design, Visualization methods and techniques.

\section{Introduction and Motivation}

Visualization enables scientists to transform data in its raw form to a visual form that will facilitate discoveries and insights. It has also been shown that there are advantages for analysis to displaying inherently 3-dimensional (3D) data, such as LiDAR data, geo-spatial data, and volumetric data, in 3D rather more difficult to see in $2 \mathrm{D}[16,17,28]$ Furthermore, displaying these $3 \mathrm{D}$ visualizations in immersive environments has additional advantages, such as additional depth information and spatial relationships. Researchers have demonstrated this in a number of applications, such as 3D seismic data [7], oil and gas exploration [6], and geoscience [16,17]. It has been also shown that understanding, discovery and scientific workflows can be also enhanced through immersion $[10,17]$. However, static visual information is not enough. Data selection and exploration is identified as a critical aspect of making discoveries from visualizations. Previous research has shown that interaction fosters analysis and discovery [21,22]. Yet, interaction is still identified as one of the areas that is in need of focused study. The 2006 NIH/NSF Visualization Research Challenges Report identifies interaction fosters analysis and discovery, yet is it one of the challenges needing more research in the context of visualization [14]. In immersive visualizations, volumes of data that the user may select are likely not to be pre-determined individual colored objects or point clouds may be displayed representing each data point, where 
a user may wish to select many of these points. Although there are some effective ways to interact with such visualizations, these techniques may require additional training. As a result, advantages provided by immersion may be hampered by the lack of suitable interaction for immersive visualizations. We have now reached the stage in which user interaction tools need to be designed, evaluated, and established specifically for immersive visualizations.

One type of interaction that we will focus on is called selection, where a user identifies an area for analysis. Many selection methods have been designed with the assumption that the areas, which the user may select, are volumes predetermined by underlying model. For example, if selecting an object with a predefined volume, such as a table, one can select at least one point on the surface mesh of that model and as a result the rest of the points defining that mesh can be associated with that selection. An example of this is 'Ray-Casting', a selection technique in which a ray is cast from the users input device out into 3D space in order to make the selection [4]. The first point that the ray intersects is the selected point. However, the selected point is usually a part of a defined surface mesh. Therefore, the entire surface mesh and associated object is selected. These types of selection techniques work well for these types of volumes in order to reduce effort needed to select each individual point of the volume. The mesh may make up a volume, but the individual points of that volume are not individually selected. $3 \mathrm{D}$ data points visualized in an environment may not have a predefined mesh that consists of a set of points. These data points may have a variety of related or not related features, but may not make up a volumetric mesh. How those points connect or relate to each other as a volume of data may not be predetermined. Therefore, there is a need for the user to be able to define the volume of data the user wishes to select. Individual colored objects or point clouds may be displayed representing each data point, where a user may wish to select a subset of these points, whether within a particular range of parameters or not. Furthermore, interacting with a visualization 3D data point clouds, consisting of thousands or millions of data points can be particularly difficult due to the density of the data, occlusion, opacity variations, and limited color scheme of features.

Although there are a few selection methods that allow users to interact with multiple points or filtering techniques, they do not necessarily allow the user to directly define the volume which users visually want to select. Picking enables users to choose individual points, but can be time consuming and tedious if the user wishes to select larger sets of points [35]. Filtering techniques, such as brushing, enables users to select larger sets of points, but the problem with these techniques is that the user needs to be searching for points that fit within a particular range of parameters $[2,13]$. At times, scientific workflow is best supported through exploration and direct selection. As a result, advantages provided by immersion may be hampered by the lack of suitable interaction techniques for immersive visualizations. There is the need for accurate, efficient, low fatigue, volumetric selection techniques for immersive visualizations, or other similar types of selection in an immersive environment. This paper focuses on detailing a classification of selection interaction for volumetric data. Additionally this paper discusses the current and future research challenges in human-computer interaction for immersive visualizations. Through a preliminary study, we found that users 
often intend to reach for or point to particular data of initial interest. Also in an initial preliminary study we found that users tend to bring the data closer to view and orient the data prior to performing other tasks. Other deductions are revolves around strategic choices based on type of user.

For volumetric selection, preliminary work identified several components to for bimanual interaction: a) defining the volume for selection b) manipulating the volume c) task assignment d) mapping function and e) manipulation function. This work was designed with the assumption that the volume for selecting is concrete. A limitation of this work are that one of two situations occur: either the user is not able to select all of the areas that is desired or that areas not desired are selected because there is only one way to select all of the desired areas. We extend this classification further to apply to more generalizable volumetric selection and address the limitations of previous work. This paper presents this extended, more detailed, classification of actions for selection interaction of volumetric data. This work can assist with the design and development of volumetric selection techniques for visualizations of 3D scientific data. Additionally, this paper demonstrates use this classification for techniques which extend across multiple platforms. And finally, the paper outlines research challenges in the context of defining selection techniques for volumetric data.

\section{Background and Related Work}

\subsection{Volumetric Data}

Volume data consist of 3D (possibly time-varying) spatially-located positions which contain information about that point. Information can consist of one to many measurable features associated with this $3 \mathrm{D}$ position, such as velocity, temperature, pressure, etc. They may not consist of tangible surfaces and edges. Information is obtained through sampling real world information or through computer generated simulations. The visual complexity increases with increase of number, density, coloring, occlusion and other properties of data points. Data points within the volume often are defined as a voxel, having $\mathrm{x}, \mathrm{y}, \mathrm{z}$ position and other attributes per which the data point is colored, and other visual aspects are defined, such as opacity, etc. Volume data is typically is inherently 3D spatial, however in some cases other types of data may be scaled down or scaled up to a 3D spatial context. An example of a visualization of volumetric data is shown in Figure 1.

\subsection{Volumetric Visualizations}

Volume visualizations are developed as a means of gaining perspective or meaningful information from these types of data using graphics and rendering for representation, interaction and manipulation [11]. Volume rendering or direct volume rendering is the process of creating an image from the extracted information derived from 3D volumetric data [11]. Usually visual representation, or coloring of, each data point is generated based on one of those features. In visualizations that display 3D volumetric data, the objects are rendered with splat-based, illustrative, or other rendering 
techniques such that the volumetric areas are defined by clouds of color with varying opacity, or by some other representation $[1,12,15]$. Using these rendering techniques an underlying mesh may not always define the relationships between each point sample. 3D volumetric visualizations can be difficult to interact with due to these properties of volumetric data and the various rendering types used in the visualizations [15].

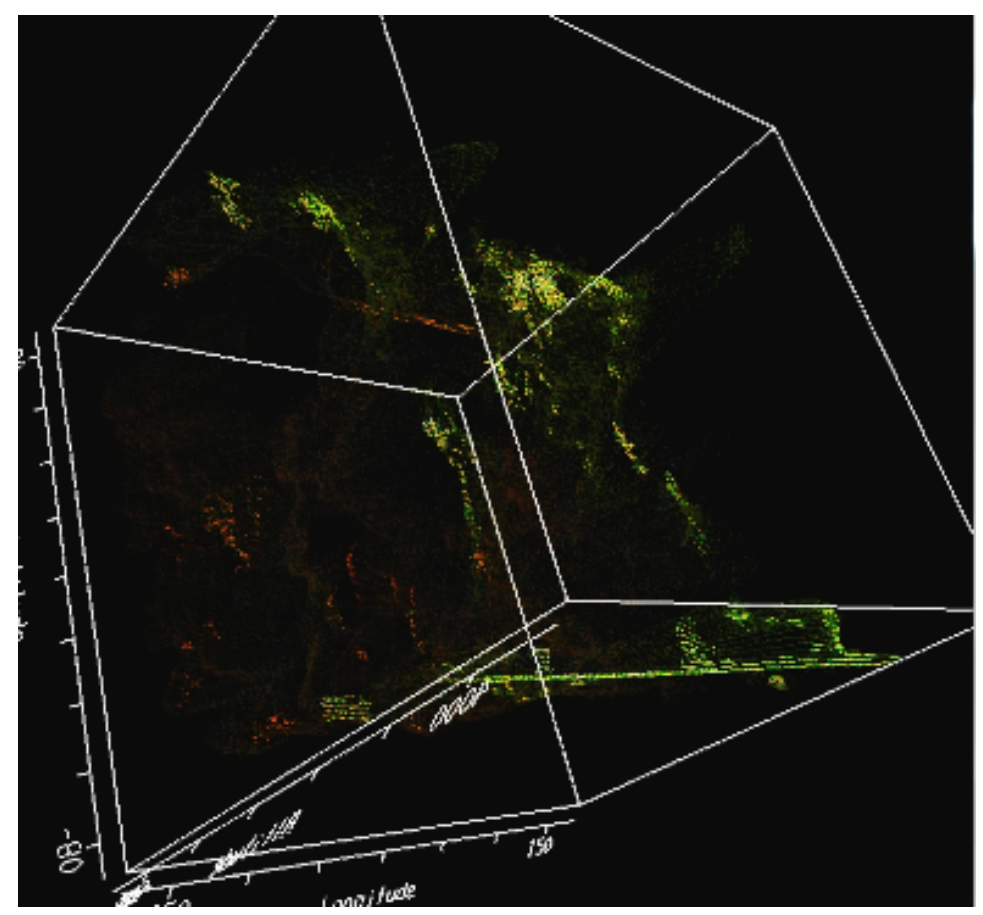

Fig. 1. Example of Visualization of Volumetric Data

\subsection{Interaction with Volumetric Visualizations}

The visual complexity and complex relationships of 3D volumetric data makes the development of effective 3D interaction techniques particularly challenging. Interaction classifications and guidelines have been developed for 3D polygonal data, or data which consists of points that define a polygonal mesh [4,32]. These papers outline taxonomies for the interaction and evaluation frameworks for testing the performance of the techniques. Designers can use the performance results of these studies to help guide them on decisions in designing interaction techniques for optimal performance. However, these guidelines and techniques for selection have been typically based on the assumption that the mesh defines the relationship between the points [27]. Scientific workflow, exploration, and analysis can be enhanced through appropriate design and evaluation of interaction and user interfaces. Although this has been completed some in previous work [4], there has been little work completed to develop guidelines 
specifically for volumetric visualizations. The following outlines initial design of selection techniques for volumetric data. Ray-casting methods adapted for volumetric displays were found to provide better selection than a 3D-point cursor when tasked with selecting multiple individual targets [8]. Lasso techniques have been developed for multiple object selection $[15,9,25,26]$. A few of these have integrated refinement capabilities. However these bodies of work only evaluated selection of individual targets rather than volumes or regions of data.

\subsection{Interaction with Immersive Volumetric Visualizations}

Recently interaction techniques have been developed and evaluated for 3D volumetric data $[8,9]$. Conic selection techniques were designed for interaction with polygonal data but can be used for volume selection [23]. In these techniques a ray is cast out into space where the ray defines a central spline where the radius of a cone increases out from that spline as the ray continues into space. Rectangular volume or region selection techniques have been developed specifically for volumetric data selection, but more thoroughly evaluated for bimanual control [29]. The results revealed that an asymmetric-synchronous selection technique is best for potentially long periods of time and for cognitively demanding tasks. However when optimum accuracy is needed, a bimanual symmetric-synchronous technique was best for selection. Another study found that asynchronous actions increased cognitive demand in asymmetric techniques [30].The limitations of these previous works are that they lack the creation of other types of volumes, such as convex volumes, or more organic. Initial techniques have been developed that are more focused on more dynamic or organic selection for volumetric data. A technique, CloudLasso, was developed to select organic shaped regions through use of 2-degrees of freedom gestures. Using the 2D gestures to define the outline of a region overlaid the visual display of data, the remaining of the volume was selected using a systematic algorithm of Marching Cubes, where threshold of density determined if a point should be in the selected set or not [34]. Additionally, another technique, called the Volume Cracker, was developed to use hand gestures to slice open volumes of data and found it to be better for exploration tasks than a standard desktop technique [3] Some work has been completed to help improve selection for immersive volumetric data, there is a need for guidelines and classification of techniques from the human-computer interaction community to better allow visualization experts and scientists to benefit from the appropriate interaction. The potential is for improved scientific workflow, exploration and analysis leading to improved discoveries.

\section{Classification}

\subsection{Preliminary Work}

Some have classified types of interaction in other types of visualizations such as "select: marking something of interest, explore: showing me something else, reconfigure: 
show me a different arrangement, encode: show me a different representation, abstract/ elaborate: show me more or less detail, filter: show me something conditionally, and connect: show me related items" [33]. Little work has been conducted to actually classify and evaluate interaction guidelines for 3D volumetric data. This paper specifically focuses on selection. An initial taxonomy of volumetric selection has been defined [31] as means to classify interaction components for volumetric selection. The taxonomy outlines the basic components of volumetric selection as: a) defining the volume for selection b) manipulating the volume c) task assignment d) mapping function and e) manipulation function. Defining the volume was limited to identifying a particular base shape, such as a sphere, a cube, a lasso, or other. Manipulating the volume include translation, rotation, and scaling of the selection volume. Task assignment and functions for manipulation and mapping refer to how each of the definition and manipulation components are assigned to the input.

\subsection{Data Collection}

We set out to expand on the preliminary classification work, and wanted to learn more about what users were inclined or innately felt how they would identify volumes of data. The purpose of this work was to determine what were the more natural actions that a user would perform as a way to expand the classification with an emphasis on user intuitiveness and user-centric design.

Study Design. We designed an initial study to collect data on how individuals would want to explore and select regions of interest based on real world actions. To simulate a set of volumetric data, physical cotton balls and stretched cotton were used as target regions for selection. Target areas were colored and other regions were not. The participants' task was to use any way possible to let the system know which regions they wanted to select (which were the colored target regions). The means of completing this task was intentionally left open-ended so that participants could describe any imaginable tool, perform any action, or tell the facilitator how they would like to complete their task. Position and orientation data was collected on physical actions through tracked hand movements. Observational notes were made by one facilitator. Audio was recorded and additional notes were taken by another observer in order to record the comments by the participants. Video recorded how the target data and other data were manipulated. The main goal was to take a user-centric approach in collecting information about designing interaction for this type of task. Instead of limiting the user to a specific set of methodology, a more open-ended approach allowed for exploration of the users wanted. It was set up this way so that we could study more about users' intentions for selection and how their actions were spatially related to the volume of data. 

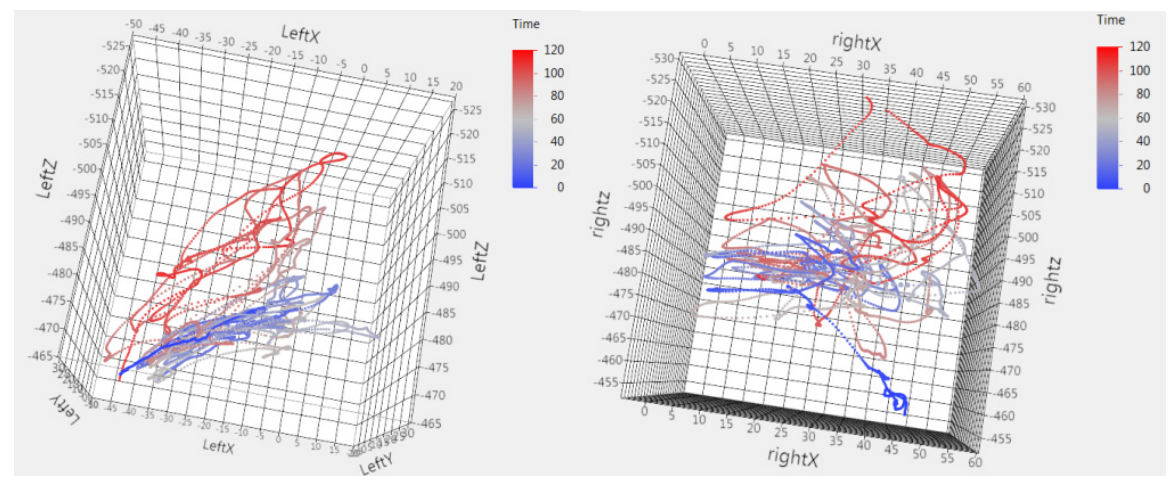

Fig. 2. Top-down view of $3 \mathrm{D}$ Scatterplot of left and right hand movements of participants, colored by time

Results. Data was collected on 10 participants. These participants were a mix of novice $(\mathrm{N}=4)$ and expert $(\mathrm{N}=6)$ users. Quantitative and qualitative data was collected. We limit the data presented to those which support our classification scheme presented in this particular paper.

Quantitative Data. Quantitative data included the position of the left and right hands as the participants performed their task. This data revealed that participants strategized their actions for volume definition over time (Figure 2). Any portions of the volume close to the user, were defined first. Defining volumes which were further from reach and out of sight, or occluded, were defined in the later portion of the time sequence. Using this data, we can classify actions of volume definition into portions actually defined by the properties of the volume itself, such as occlusion and proximity to user. Quantitative data was also used to break down larger groups of actions into subsets of actions. Actions can be broken down into initial volume definition and then later a refinement step to that volume. Actions can also be broken down into defining the volume, adjusting the view or manipulating the data to have a better advantage for selection tasks, and strategizing. Strategizing involves users studying the volume for features about what to select and alternatively about how to select it.

Qualitative Data. Observational data revealed that users often intend to physically reach for or point to particular data of initial interest, but then bring it closer for more actions to that task. We can classify these actions as change in context, anything which users will to do change their view or to bring data closer to them for more detailed interaction. Users also tended to bring the data closer to view and change the orientation of the data prior to indication of selection. Quantitative data collected supports these observational action sequences. This can also be interpreted that, before any selection, there is an observational or exploration step. Users are strategizing the best way to identify their region of interest. Strategizing can be based on time (ie. what is seen first, may be selected), or overall volumetric region of data (all data is seen, but what is the most efficient way to identify that volume). 
Data Based on User Type. Differences between expert and novice users were as follows. Quantitative data indicated that expert users were quicker ( $M=70 \mathrm{sec}$, $\mathrm{SD}=1.34$ ) in their decision-making or strategizing processes. Novice users had more delay between actions, with total time being longer $(M=120 \mathrm{sec}, \mathrm{SD}=2.45)$ indicating they were thinking for longer periods of time in order to determine their course of actions to select the volume. Qualitative data revealed that expert users were more interested in how to explore the data. Often expert users move other data away from the target data to have more space around the target data to be able to see, explore, and select it. Novice users were more interested in completing the task as accurately as possible. This could attribute to why it took them longer as well.

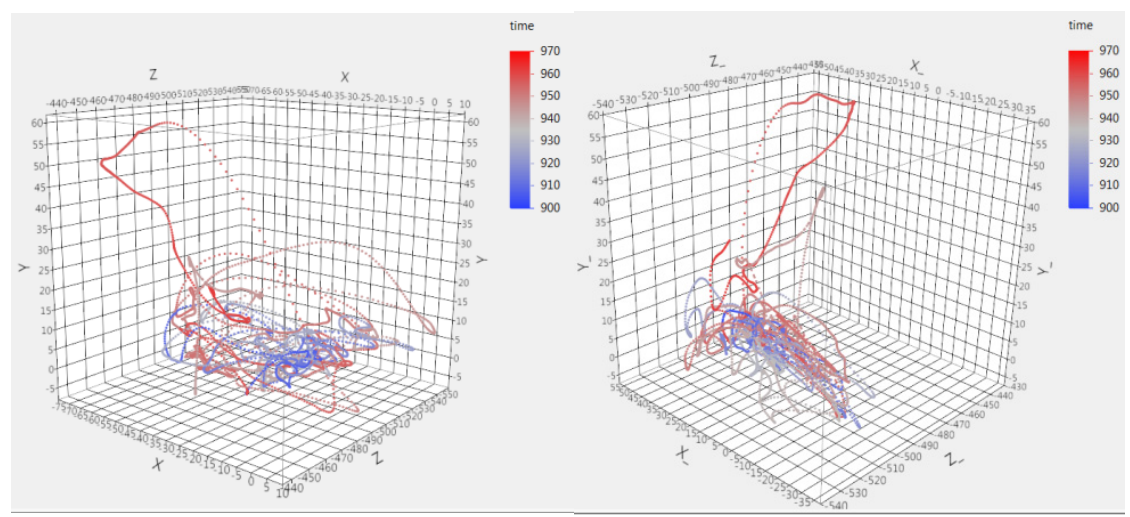

Fig. 3. 3D scatterplot showing reach actions to bring data within closer proxmmitity to the user

\subsection{Discussion of Classification Extension and Challenges}

We present extensions of the current classification for volumetric selection: a) selection volume definition b) selection volume manipulation c) task assignment d) mapping function and e) manipulation function [31], discussed in section 3.1. These guidelines as described in this classification, may be extended across spatial dimensionality, display type, and platforms. This classification can be used as a means for designing new selection techniques for volumetric data to ensure appropriate functionality and components are present for the user.

Defining Volume Selection Sets. There are two ways to classify definition of the volume: additive or subtractive. In an additive technique, users can identify data points or volumes of data points to add to the selection set. To remove them, they identify them again to switch them from being in the set to out of the set. In a subtractive technique, a user identifies all of the data which they do not want selected. Any data that is not identified will be included in the selection set. To remove data from the selected set, users will then identify those data, and as a result, remove them from the selected set. The challenges coupled with definition are occlusion, blending 
issues, data too densely populated, etc. To help with this challenge, implementing techniques which account for user-system symbiotic actions (Section 3.3.3) or data manipulation (Section 3.3.4) of the extended classification. Furthermore, methods which can provide more organic or specific spine patterns to encompass the data may be useful.

Refining the Volume Selection Sets. From previously designed techniques and our presented data, what is missing in the two sections, of defining and manipulating the volume, are methods to refine the volume, such as adding or removing data from the selected volume. Volumetric selection techniques should have a means by which to edit the volume of the selected data points. Additive and subtractive methods may also be used to modify the existing set of selected data points. However, other methods may include a means of manipulating the volume itself for refinement. It may be important to keep definition, manipulation, and refinement of the volume as separate interaction functionalities as there may be challenges if the methods chosen are not best-suited to the particular task.

User-System Symbiotic Actions. Also what is missing from the classification are adaptations to account for properties of the technique, such as large-scale data, such as those portions of the data out of reach, or with multiple dimensions, such as time series data. Such methods may include automation, such as in Yu's Lasso technique [34], where the system augments the user's action with its own attempt at refining the volume selection set. This augmentation is a form of man-computer symbiosis [20]. Except in this sense, we concept is modified such that the 3D UI can harness the strengths of a user and augment them with strengths of the system, to work in tandem to select and explore the data. A challenge with this aspect of the classification is that there is not a well-defined line when to engage the system and when to harness the user's capabilities. Some of this may be domain-specific, however it is important when designing to consider the trade-offs between the two. Also one may confuse this with system tools. The main idea to keep in mind is to maintain this idea of a partnership, where the system picks up where the user left off and the user picks up where the system left it.

Data Manipulation: Extension of Self or Retraction of Data. Another adaptation to this would allow for extension of selection techniques to move beyond reach of the user, or provide functionality to bring the data closer to them, as we concluded from our collected data. The data itself can be manipulated, such as decoupled as in [3], scaled, deformed, etc. This may allow for a perspective on the data that can permit a better selection. An important aspect to remember for visualizations is that when designing interaction techniques, the data itself needs to maintain its relative scale and relationships. Manipulation of the data will permit the user to bring data closer, but techniques need to either retain the relationships or allow the user to revert back to them once completed with interacting with the data in its modified form. The challenge with this aspect is determining or providing the flexibility to decide when to 
manipulate the view as compared with when to manipulate the data. There are advantages and disadvantages to either that are particularly domain-specific.

Strategy Driven. Techniques that either adapt to the user or learn from time intervals between actions can be used to enhance the user's completion of selection. For example, if a user is delayed, it might mean that they are either a novice user, and are unsure what to do, or are an expert user, so suggesting elements of selection might be of use. Implementing in the system a way to learn the users strategies can be helpful to providing more insight and detracting from the user interface itself. The challenge here is how to determine what the users' intention really was or ultimately learning from those strategies. Other input mechanisms and data collected can assist to help determine users strategies as other ways to help determine intent and augment the discovery process.

\section{Conclusion and Future Work}

This paper presented some initial research work on more what users are more inclined to or innately felt how they would identify volumes of data. Through quantities and qualitative analysis, an extension to an existing classification was provided to serve as a way to design future volumetric interaction techniques. In conclusion the extensions to the classification include: more dynamic and organic ways to define the volume selection set, methods for refining the volume selection set, user-system symbiotic actions and functionality, data manipulation, and strategy driven. The purpose of this work was to determine what were the more natural actions that a user would perform as a way to expand the classification with an emphasis on user intuitiveness and usercentric design. Each were based on previous and preliminary work of data collected of users actions. Each section provided description, examples, and challenges to consider. In the future, we will implement these concepts into design and provide benchmarks for evaluations of volumetric interaction techniques.

Acknowledgements. Neera Pradhan and Anh Nguyen were instrumental for writing code for the software to collect the data. Neera Pradhan and Zhibo Sun helped to perform initial collection of the data for this work. A special thanks is extended to them for their efforts.

\section{References}

1. Ayala, D., Pla, N., Vigo, M.: Splat representation of parametric surfaces. Computing 79, 101-108 (2007)

2. Becker, R.A., Cleveland, W.S.: Brushing scatterplots. Technometrics 29(2), 127-142 (1987)

3. Laha, B., Bowman, D.A.: Volume cracker: a bimanual 3D interaction technique for analysis of raw volumetric data. In: Proceedings of the 1st Symposium on Spatial User Interaction (SUI 2013), pp. 61-68. ACM, New York (2013) 
4. Bowman, D., Kruijff, E., LaViola, J., Poupyrev, I.: 3D User Interfaces: Theory and Practice. Addison-Wesley, Boston (2004)

5. Elmqvist, N., Dragicevic, P., Fekete, J.: Rolling the Dice: Multidimensional Visual Exploration using Scatterplot Matrix Navigation. IEEE Transactions on Visualization and Computer Graphics 14(6), 1141-1148 (2008)

6. Evans, F., Volz, W., Dorn, G., Frohlich, B., Roberts, D.M.: Future trends in oil and gas visualization. In: VIS 2002: Proceedings of the Conference on Visualization 2002, pp. 5562. IEEE Computer Society, Washington, DC (2002)

7. Frohlich, B., Barrass, S., Zehner, B., Plate, J., Gobel, M.: Exploring geo-scientific data in virtual environments. In: Proceedings of IEEE Visualization, pp. 169-173 (1999)

8. Grossman, T., Balakrishnan, R.: The design and evaluation of selection techniques for 3D volumetric displays. In: UIST 2006, pp. 3-12. ACM Press (2006)

9. Grossman, T., Wigdor, D., Balakrishnan, R.: Multi-finger gestural interaction with $3 \mathrm{~d}$ volumetric displays. In: UIST 2004, pp. 61-70. ACM Press (2004)

10. Gruchalla, K.: Immersive well-path editing: investigating the added value of immersion. IEEE Virtual Reality, 157-164 (2004)

11. Hansen, C.D., Johnson, C.R. (eds.): The Visualization Handbook, p. 120. Elsevier (2005)

12. Jang, J., Ribarsky, W., Shaw, C.D., Faust, N.: View-Dependent Multiresolution Splatting of Non-Uniform Data. In: Proceedings of the Symposium on Data Visualization. ACM International Conference Proceeding Series, vol. 22, pp. 125-ff (2002)

13. Janicke, H., Bottinger, M., Scheuermann, G.: Brushing of attribute clouds for the visualization of multivariate data. IEEE Transactions on Visualization and Computer Graphics 14(6), 1459-1466 (2008)

14. Johnson, C., Moorhead, R., Munzner, T., Pfister, H., Rheingans, P., Yoo, T.S.: NIH/NSF Visualization Research Challenges Report. IEEE Press (2006)

15. Kalaiah, A., Varshney, A.: Modeling and Rendering of Points with Local Geometry. IEEE Transactions on Visualization and Computer Graphics 9(1), 30-42 (2003)

16. Kreylos, O., Bawden, G., Bernardin, T., Billen, M., Cowgill, E., Gold, R., Hamann, B., Jadamec, M., Kellogg, L., Staadt, O., Sumner, D.: Enabling scientific workflows in virtual reality. In: Proceedings of the 2006 ACM International Conference on Virtual Reality Continuum and its Applications, VRCIA 2006, pp. 155-162. ACM, New York (2006)

17. Kreylos, O.: Environment-Independent VR Development. In: Bebis, G., et al. (eds.) ISVC 2008, Part I. LNCS, vol. 5358, pp. 901-912. Springer, Heidelberg (2008)

18. Kreylos, O., Bawden, G.W., Kellogg, L.H.: Immersive visualization and analysis of LiDAR data. In: Bebis, G., et al. (eds.) ISVC 2008, Part I. LNCS, vol. 5358, pp. 846-855. Springer, Heidelberg (2008)

19. Kopper, R., Bacim, F., Bowman, D.A.: Rapid and Accurate 3D Selection by Progressive Refinement. In: Proc. 3DUI, pp. 67-74. IEEE Computer Society, Los Alamitos (2011)

20. Licklider, J.C.R.: Man-computer symbiosis. IRE Transactions on Human Factors in Electronics (1), 4-11 (1960)

21. Lin, C., Loftin, R., Stark, T.: Virtual reality for geosciences visualization. In: Proceedings of 3rd Asia Pacific Computer Human Interaction Conference, pp. 196-201 (1998)

22. Lin, C., Loftin, R., Nelson, J.: Interaction with geoscience data in an immersive environment. In: Proceedings of IEEE Virtual Reality, pp. 55-62 (2000)

23. Liang, J., Green, M.: JDCAD: A Highly Interactive 3D Modeling System. Computers \& Graphics 18(4), 499-506 (1994)

24. Yu, L., Efstathiou, K., Isenberg, P., Isenberg, T.: Efficient Structure-Aware Selection Techniques for 3D Point Cloud Visualizations with 2DOF Input. IEEE Transactions on Visualization and Computer Graphics 18(12), 2245-2254 (2012) 
25. Lucas, J.F., Bowman, D.A.: Design and Evaluation of 3D Multiple Object Selection Techniques. Report, Virginia Polytechnic Institute and State University, USA (2005)

26. Olwal, A., Feiner, S.: The flexible pointer- An interaction technique for selection in augmented and virtual reality. In: UIST 2003, pp. 81-82 (2003)

27. Sherman, W.R., Craig, A.B.: Understanding Virtual Reality: Interface, Application, and Design. Morgan Kaufmann Publishers Inc. (2002)

28. Tory, M., Kirkpatrick, A., Atkins, M.S., Moller, T.: Visualization Task Performance with 2D, 3D, and Combination Displays. IEEE Transactions on Visualization and Computer Graphics 12(1), 2-13 (2006)

29. Ulinski, A., Wartell, Z., Hodges, L.F.: Bimanual Task Division Preferences for Volume Selection. In: Spencer, S.N. (ed.) Proceedings of the 2007 ACM Symposium on Virtual Reality Software and Technology, VRST 2007, pp. 217-218. ACM, New York (2007)

30. Ulinski, A., Zanbaka, C., Wartell, Z., Goolkasian, P., Hodges, L.F.: Two Handed Selection Techniques for Volumetric Data. In: Proceedings of the 3D User Interfaces, 3DUI 2007, pp. 107-114. IEEE Computer Society (2007)

31. Ulinski, A.: Ph.D. Dissertation, UNC-Charlotte (2008)

32. Ware, C., Rose, J.: Rotating virtual objects with real handles. ACM Trans. Comput.-Hum. Interact. 6, 162-180 (1999)

33. Yi, J.S., Kang, Y., Stasko, J.T., Jacko, J.A.: Toward a Deeper Understanding of the Role of Interaction in Information Visualization. In: Proceedings of InfoVis 2007, vol. 13, pp. 1224-1231 (2007)

34. Yu, L., Efstathiou, K., Isenberg, P., Isenberg, T.: Efficient Structure-Aware Selection Techniques for 3D Point Cloud Visualizations with 2DOF Input. IEEE Transactions on Visualization and Computer Graphics 18(12), 2245-2254 (2012)

35. Zhang, J.H., Liang, C., Li, G.Q.: 3D Primitive Picking on GPU. Journal of Engineering Graphics 1, 10 (2009) 